\title{
Using computerized tomography perfusion to measure cerebral hemodynamics following treatment of traumatic brain injury in rabbits
}

\author{
KEFEI CHEN, FEIHU DAI, GUANGXU LI, JIRONG DONG and YUHAI WANG

\begin{abstract}
Department of Neurosurgery, Wuxi Clinical College of Anhui Medical University, Craniocerebral Injury Cure Center of People's Liberation Army, The 101st Hospital of People's Liberation Army, Wuxi, Jiangsu 214044, P.R. China
\end{abstract}

Received November 1, 2018; Accepted May 16, 2019

DOI: $10.3892 /$ etm.2019.7785

\begin{abstract}
The present study aimed to investigate the use of computerized tomography (CT) perfusion for evaluating cerebral hemodynamics following traumatic brain injury (TBI) in rabbits. The animals were randomly assigned into four groups ( $n=10$ animals/group): i) Control, ii) TBI, iii) TBI + common decompression and iv) TBI + controlled decompression groups. A TBI model was established in rabbits using epidural balloon inflation. In the groups receiving intervention, animals were provided common decompression or controlled decompression treatments. Conventional CT and CT perfusion scanning were performed, with cerebral hemodynamic indices, including regional cerebral blood flow (rCBF), regional cerebral blood volume (rCBV) and mean transit time (MTT) being measured. Blood-brain barrier (BBB) permeability was evaluated using Evans blue staining. Compared with those in the control group, $\mathrm{rCBF}$ and $\mathrm{rCBV}$ values of the bilateral temporal lobes and basal ganglion in the TBI, TBI + common decompression and TBI + controlled decompression groups were significantly lower, whereas the MTT values were markedly prolonged and Evans blue dye content was greatly increased $(\mathrm{P}<0.01)$. Controlled decompression was demonstrated to be more potent than common decompression for preventing TBI-induced decline in $\mathrm{rCBF}$ and $\mathrm{rCBV}$ values in the bilateral temporal lobes and basal ganglion, as well as reversing TBI-induced extension of MTT in the bilateral temporal lobes $(\mathrm{P}<0.01$ vs. TBI group). However, neither common nor controlled decompression could reduce TBI-induced increase in BBB permeability. In conclusion, these findings indicate that CT perfusion may be used
\end{abstract}

Correspondence to: Dr Yuhai Wang, Department of Neurosurgery, Wuxi Clinical College of Anhui Medical University, Craniocerebral Injury Cure Center of People's Liberation Army, The 101st Hospital of People's Liberation Army, 101 Xingyuan North Road, Wuxi, Jiangsu 214044, P.R. China

E-mail: wangyuhai_pla@163.com

Key words: computed tomography perfusion, traumatic brain injury, cerebral hemodynamics, compression, decompression to monitor cerebral hemodynamics following TBI in rabbits. Controlled decompression was deduced to be more potent than common decompression for preventing abnormalities in cerebral hemodynamics after TBI.

\section{Introduction}

Severe traumatic brain injury (TBI) is a leading cause of death and disability, which puts a heavy strain on the global economy (1). It is reported that $\sim 5.48$ million cases of TBI occur annually worldwide, most of which are associated with road traffic injuries (1). TBI is classified into mild [Glasgow Coma Scale (GCS score), 13-15], moderate (GCS score, 9-12) and severe (GCS score, $\leq 8)$ TBI based on disease severity $(2,3)$. The survival and therapeutic outcomes for severe TBI are extremely poor. According to statistical data of the Chinese population, in 7,145 cases of acute head trauma, the mortality rate for severe TBI stood at $21.8 \%$ (4). In addition, several factors have been reported to correlate with the outcome of severe TBI, including intracranial pressure (ICP) (4-6), brain hypoxia (5), cerebral perfusion pressure (5) and quantitative cerebral blood flow within $12 \mathrm{~h}$ following injury (7).

The diagnosis, treatment and prognosis prediction of TBI is performed using a number of non-invasive neuroimaging methods, including conventional non-contrast computerized tomography (CT), CT perfusion, positron emission tomography and perfusion magnetic resonance imaging $(8,9)$. However, the accuracy and precision of conventional CT is not satisfactory (10). In relation to cerebral injuries and hemodynamic changes before and after treatment, CT perfusion provides increased sensitivity and more valuable information compared with conventional non-contrast CT (11). Additionally, more specific information regarding circulatory disturbances, including regional cerebral blood flow ( $\mathrm{rCBF})$, regional cerebral blood volume (rCBV) and mean transit time (MTT), can also be obtained using CT perfusion (12). However, data regarding cerebral hemodynamics following severe TBI have yet to be characterized using perfusion $\mathrm{CT}$.

Accumulating evidence indicates that early cranial decompression is a potential strategy for improving survival and preventing disability in patients afflicted with severe TBI (13-16). Cranial decompression supports the reduction 
of ICP and is generally associated with favorable clinical outcomes in patients with TB; however, the precise role of cranial decompression in the treatment of TBI remains unclear, as it also has been reported that decompression may result in unfavorable outcomes and disabilities (17). In addition, the effects of decompression on the cerebral hemodynamic changes after TBI have yet to be fully described.

In the present study, cerebral hemodynamics in a rabbit model of severe TBI induction was examined using CT perfusion. In particular, the therapeutic outcomes following treatment with either common or controlled decompression according to cerebral hemodynamic changes were also investigated using this technique. The findings presented in this study may provide valuable insights into the use of CT perfusion to understand and interpret intervention outcomes for severe TBI.

\section{Materials and methods}

Reagents. Ketamine was obtained from Jiangsu Hengrui Medicine Co., Ltd. Droperidol was purchased from Shanghai Xudong Haipu Pharmaceutical Co., Ltd. Iopromide 370 was purchased from Bayer AG. Evans blue dye was purchased from Sigma-Aldrich (Merck KGaA).

Animals and experimental assignment. A total of 20 male and 20 female adult healthy New Zealand white rabbits (weight, 2.5-3.0 kg; age, 7 months) were obtained from the Laboratory Animal Center, Jiangsu Institute of Parasitic Diseases [Wuxi, China; license no. for use of experimental animals: SXYK (Su) 2015-0023]. Animals were housed in a temperature- $\left(\sim 25^{\circ} \mathrm{C}\right)$ and humidity- (50-60\%) controlled room maintained on a 10:14-h light-dark cycle with free access to food and water. Animals were randomly assigned into four groups $(n=10$ rabbits/group): i) Control, ii) TBI, iii) TBI + common decompression or iv) TBI + controlled decompression. The present animal study was approved by the Animal Ethics Committee of Wuxi Clinical College, Anhui Medical University (Wuxi, China).

Establishment of TBI model in rabbits and interventions. To generate the TBI model in rabbits, compression was induced in animals by epidural balloon catheter inflation according to a previously performed protocol (18). Briefly, animals were given $25 \mathrm{mg} / \mathrm{kg}$ ketamine by intraperitoneal injection and $1.0 \mathrm{mg} / \mathrm{kg}$ droperidol by intramuscular injection (19). Following anesthesia, rabbits were placed on an operating table in the prone position with their heads fixed. A midline incision was made in the parietal bone to expose the skull, and an aperture $5 \mathrm{~mm}$ in diameter was made between the left interparietal bone and the midline of the parietal bone using a bone drill (Guangzhou Senxuan Medical Instrument Co., Ltd.). The dura mater was then stripped off from the inner skull plate, and a balloon catheter (Haiyan Kangyuan Medical Instrument Co., Ltd.), which is in turn connected to a TCI-II micro-infusion pump (Guangxi VERYARK Technology Co., Ltd.), was placed into the epidural space. A needle was then inserted into the aperture of the dura mater, and a Codman intracranial pressure sensor (Johnson \& Johnson) was placed into the brain tissue at a depth of $\sim 1 \mathrm{~cm}$. For TBI, approximately $0.5-1 \mathrm{ml}$ of normal saline was infused into the balloon catheter through the micro-infusion pump at a rate of $10 \mathrm{ml} / \mathrm{h}$ until the ICP value reached $25 \mathrm{mmHg}$; this infusion was sustained for $15 \mathrm{~min}$.

For the TBI + common/controlled decompression groups, the infusion of normal saline was performed as described in the TBI group. In the TBI + common decompression group, normal saline was immediately pumped out from the balloon catheter, before animals were monitored for another $15 \mathrm{~min}$. In the TBI + controlled decompression group, normal saline was slowly pumped out from the balloon catheter, stopping for $5 \mathrm{~min}$ when the ICP value had declined to 2015 and $10 \mathrm{mmHg}$ before the saline was pumped out completely. In the control group, the animals were not infused with normal saline.

At the end of all treatment procedures, all rabbits were examined using conventional $\mathrm{CT}$ scanning and $\mathrm{CT}$ perfusion scanning.

Conventional CT and CT perfusion scanning. CT scanning was performed using a 320-row spiral CT scanner (Aquilion One; Toshiba Corp.). For conventional CT scanning, rabbits under anesthesia were placed in the prone position with their heads fixed. The coronal CT images were acquired at $5 \mathrm{~mm}$ slice thickness. The parameters for CT scanning were as follows: Tube voltage, $80 \mathrm{kV}$; current, $100 \mathrm{~mA}$; matrix, $512 \times 512$ and field of view, $10 \times 10 \mathrm{~cm}$. After conventional CT scanning, CT perfusion scanning was performed at the basal ganglia. The non-ionic contrast media Iopromide $370(370 \mathrm{mgI} / \mathrm{ml}$; $1.5 \mathrm{ml} / \mathrm{kg}$ ) was injected through a binocular high-pressure injector into the ear vein at a rate of $1.0 \mathrm{ml} / \mathrm{sec}$. Normal saline was injected at the same rate. After $5 \mathrm{sec}$, dynamic CT images were acquired at $0.5-\mathrm{mm}$ slice thickness. The parameters for CT perfusion scanning were as follows: Voltage, $80 \mathrm{kV}$; current, $120 \mathrm{~mA}$; matrix, 512x512; and field of view, $10 \times 10 \mathrm{~cm}$. A total of 2,720 raw images were acquired within $35 \mathrm{sec}$.

Image processing. Following data acquisition, images were uploaded onto a GE ADW4.6 Workstation (GE Healthcare) and processed using Stroke mode in the CTP-4D software (version 11.3) of the Workstation. The cerebral middle cerebral artery and the superior sagittal sinus were automatically set as the input artery and output vein, respectively. A parametric diagram reflecting blood perfusion in cerebral tissues was constructed. The diagram was evaluated by a senior neuroradiologist, who elected each region of interest (ROI) $\sim 2 \mathrm{~mm}^{2}$ in size at the bilateral temporal lobes and basal ganglion and measured the $\mathrm{rCBF}, \mathrm{rCBV}$ and MTT. Areas were selected to avoid vessels and damaged brain tissues. The values for each parameter within the ROIs were recorded, from which the average values were calculated.

Determination of $B B B$ permeability. Animals were injected with $2 \mathrm{~g} / \mathrm{l}$ Evans blue dye $(2 \mathrm{ml} / \mathrm{kg})$ through the ear vein $1 \mathrm{~h}$ prior to sacrifice. Animals were perfused with 200-300 ml normal saline to remove Evans blue dye from the blood vessels. Brain tissues from the bilateral temporal lobes were carefully removed from the animals following sacrifice. A total of $3 \mathrm{ml}$ formamide was added to $0.5 \mathrm{~g}$ of the brain tissue homogenate to dissolve the Evans blue dye. After incubation in a $37^{\circ} \mathrm{C}$ water bath for $48 \mathrm{~h}$, the samples were centrifuged at $755 \mathrm{x} g$ for $5 \mathrm{~min}$. The supernatant was then collected before 
absorbance measurement at $632 \mathrm{~nm}$ was performed using a UV spectrophotometer (Hitachi, Ltd.). Evans blue content was calculated according to the standard curve.

Statistical analysis. Data were analyzed using IBM SPSS statistics version 23 (IBM Corp.). All experimental results are presented as the mean \pm standard deviation. Data were compared using one-way analysis of variance. In cases of variance non-homogeneity [in the $\mathrm{rCBF}, \mathrm{rCBV}$ and the MTT data in the basal ganglion $(\mathrm{P}<0.05)$ ], Welch's t-test was applied. Comparisons between groups were made using the Games-Howell post-hoc test. Variance homogeneity was detected in the MTT data of bilateral temporal lobes $(\mathrm{P}=0.245)$ and Evans blue content data $(\mathrm{P}=0.074) \mathrm{P}<0.05$ was considered to indicate a statistically significant difference.

\section{Results}

Conventional CT imaging. A clear cerebral sulcus and cerebral gyrus could be observed in the control group (Fig. 1, Control); the CT value in the frontal lobe of the white matter was calculated to be 29-30 HU. Following compression, the cerebral sulcus was absent from the CT images, and the cerebral gyrus was unclear (Fig. 1, TBI); the CT value was observed to be 25-27 HU in the TBI group. After decompression, the cerebral sulci of the bilateral hemispheres were absent from the CT scanning images; the CT values were reduced further to 18-20 HU and 20-22 HU in the TBI + common decompression and TBI + controlled decompression groups, respectively (Fig. 1, TBI + common decompression and TBI + controlled decompression).

CT perfusion imaging. The regions of interest selected for the measurement of $\mathrm{rCBF}$ in the temporal lobe and the basal ganglia in the control group is shown in Fig. 2. Compared with those in the control group, the local cerebral rCBF and rCBV values were demonstratively decreased in the TBI, $\mathrm{TBI}+$ common decompression and TBI + controlled decompression groups (Fig. 3), suggesting reduced blood flow to the rabbit brain tissues. In addition, prolonged MTTs were observed in the TBI, TBI + common decompression, and $\mathrm{TBI}+$ controlled decompression groups, with the most notable MTT extension detected in the common decompression group (Fig. 3). The time-CT value curves for the bilateral temporal lobes and the basal ganglion were also presented in Fig. 3.

Comparison of CT perfusion parameters between the four groups. The CT perfusion parameters between the four experimental groups were compared in Table I. The rCBF and rCBV values of the bilateral temporal lobes and basal ganglion in the TBI, TBI + common decompression and TBI + controlled decompression groups were significantly lower compared with the control group $(\mathrm{P}<0.01)$. Comparison between the TBI, TBI + common decompression and TBI + controlled decompression groups also revealed significant differences in the $\mathrm{rCBF}$ and $\mathrm{rCBV}$ values of the bilateral temporal lobes and basal ganglion $(\mathrm{P}<0.01)$. Notably, controlled decompression slightly alleviated TBI-induced reductions of $\mathrm{rCBF}$ and rCBV values in the bilateral temporal lobes and basal ganglion compared with TBI group $(\mathrm{P}<0.01)$. Common decompression

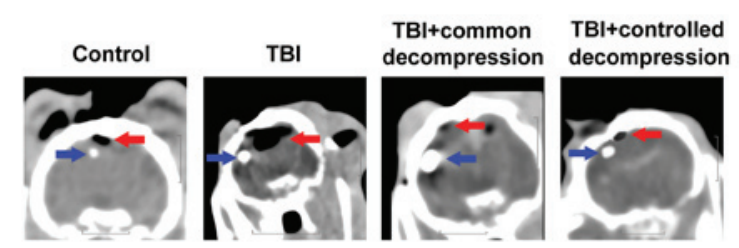

Figure 1. Representative coronal conventional CT images from rabbits in the four experimental groups. Blue arrows indicate the location of ICP monitoring probes; red arrows indicate the location of epidural balloon catheters. Scale bars, $1 \mathrm{~cm}$. CT, computerized tomography; TBI, traumatic brain injury.

only rescued the TBI-induced decrease in $\mathrm{rCBV}$ in the basal ganglion compared with TBI group $(\mathrm{P}<0.01)$ and worsened the other parameter index values ( $\mathrm{rCBF}$ of bilateral temporal lobes and basal ganglion; rCBV of bilateral temporal lobes). Compared with the control group, the MTT values of the bilateral temporal lobes and basal ganglion in the TBI, TBI + common decompression, and TBI + controlled decompression groups were significantly increased $(\mathrm{P}<0.01)$. Significant differences were also observed between the TBI and TBI + intervention groups $(\mathrm{P}<0.01)$. In particular, the TBI-induced extension of MTT in the bilateral temporal lobes appeared to be partially reversed by controlled decompression $(\mathrm{P}<0.01)$, but common decompression further prolonged the MTT values in the bilateral temporal lobes and basal ganglion compared with the TBI group $(\mathrm{P}<0.01)$.

Comparison of $B B B$ permeability between the four groups. The Evans blue dye content in the control group was significantly lower compared with the other experimental groups (Table I), representing intact BBB in the control rabbit brain. Significantly increased BBB permeability was detected in the TBI, TBI + common decompression and TBI + controlled decompression groups compared with control $(\mathrm{P}<0.01$; Table I). However, neither common decompression nor controlled decompression could reduce the increase in $\mathrm{BBB}$ permeability induced by TBI.

\section{Discussion}

The use of CT perfusion for the diagnosis and evaluation of treatment outcomes has not been widely applied. In the present study, a TBI model was developed in rabbits through epidural balloon catheter inflation. The efficacy of common decompression or controlled decompression for alleviating changes in cerebral hemodynamics induced by TBI in model animals was determined using CT perfusion. Findings in the present study demonstrated that controlled decompression contributed to improved cerebral hemodynamics compared with common decompression for TBI treatment in rabbits.

High ICP levels increase the risk of death, as a previous study reported that the mortality rate for patients with acute TBI and an ICP 20-40 mmHg is higher than that for patients with an ICP $<20 \mathrm{mmHg}$ (21.4 vs. 6.3\%) (4). In Sprague-Dawley rats, an ICP of $30 \mathrm{mmHg}$ is known as the lower threshold for cerebrovascular autoregulation (20). In a rabbit model of acute intracranial hypertension, impaired cerebral microcirculation occurs with an ICP $\geq 28.5 \mathrm{mmHg}$ (21). In addition, 

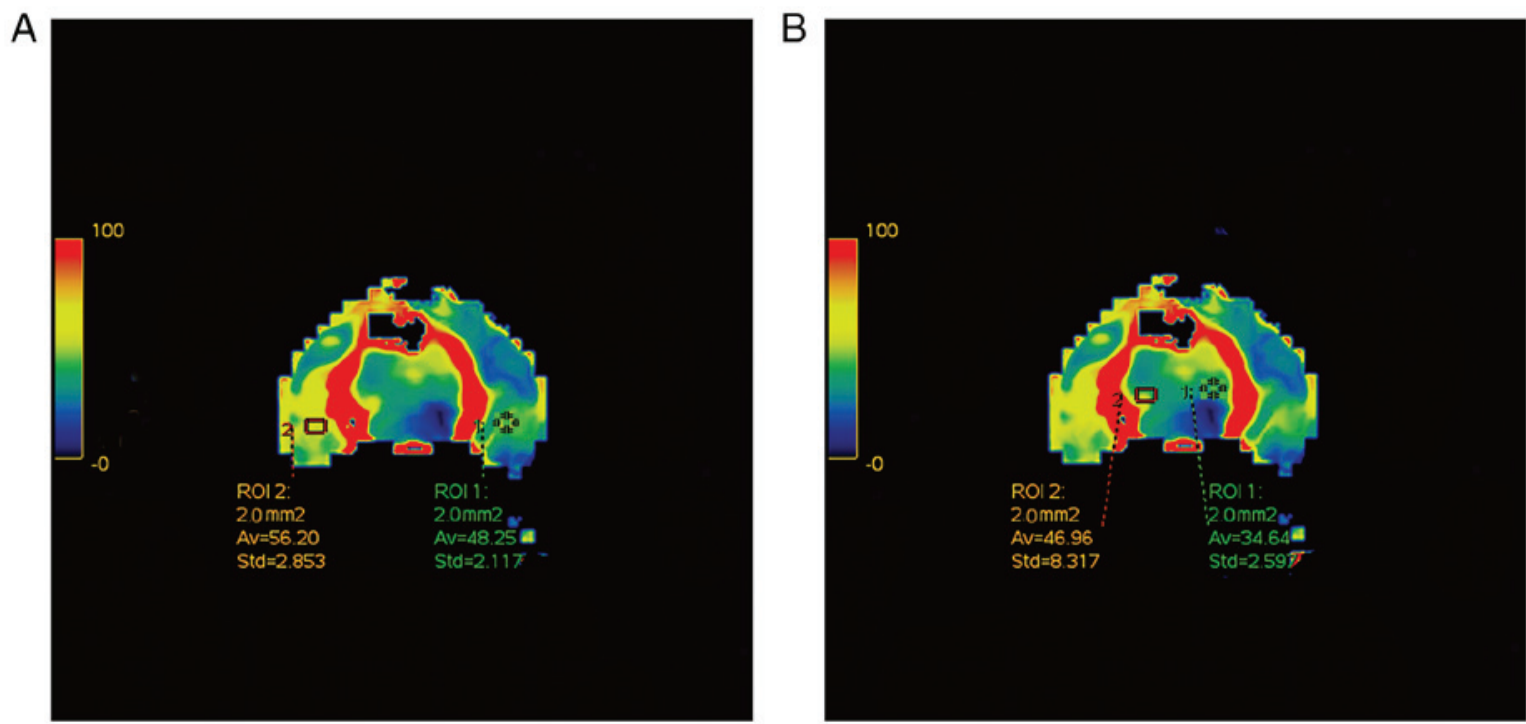

Figure 2. Measurements of rCBF in the temporal lobe and basal ganglia from the control group. (A) Temporal lobe; (B) basal ganglia. Red=good blood flow; blue=poor blood flow. rCBF, regional cerebral blood flow.
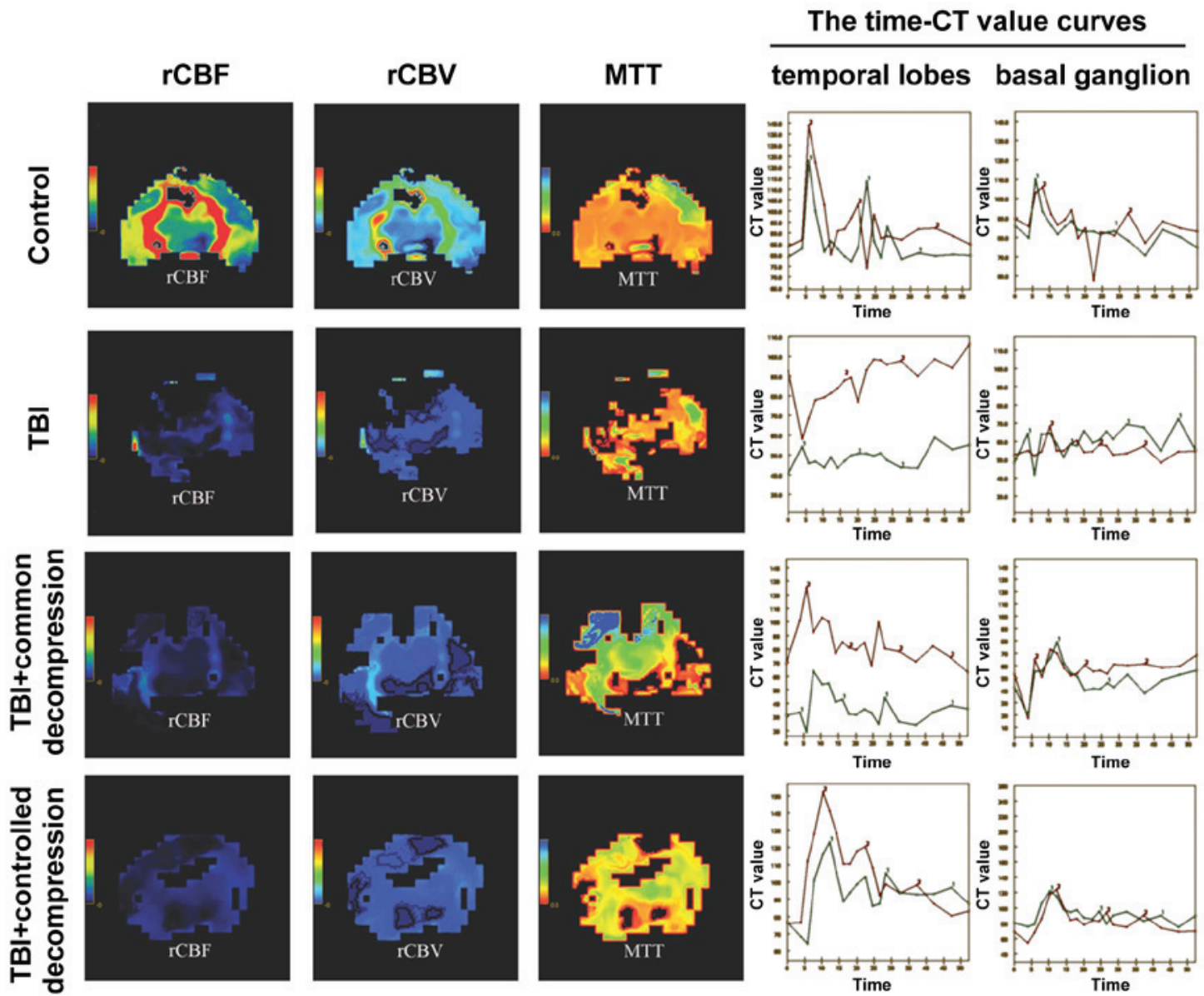

Figure 3. Representative coronal CT perfusion images from rabbits in the four experimental groups. The rCBF, rCBV, MTT and time-CT value curves for the bilateral temporal lobes as well as the basal ganglion are shown. Green and red curves represent time-CT values obtained from the left and right cerebral hemispheres, respectively. CT, computerized tomography; ICP, intracranial pressure; MTT, mean transit time; rCBF, regional cerebral blood flow; rCBV, regional cerebral blood volume; TBI, traumatic brain injury.

Donnelly et al (22) reported that cortical perfusion and vascular reactivity are significantly decreased with a $10 \mathrm{mmHg}$ increase in ICP. The baseline ICP value for healthy rabbits lie in the $5-9 \mathrm{mmHg}$ range. As a consequence of these findings, 


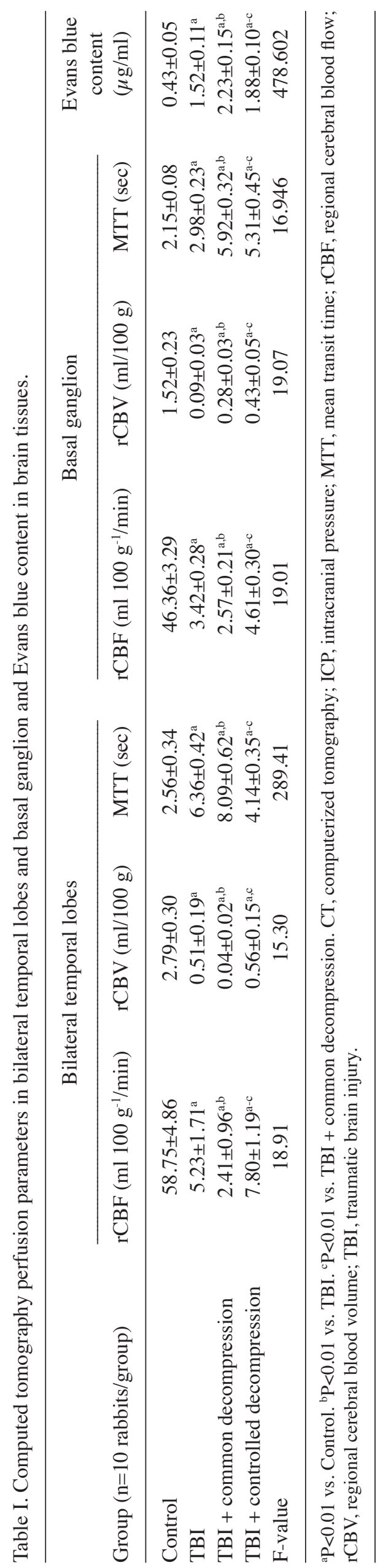

the ICP value of $25 \mathrm{mmHg}$ was elected for the present study. Indeed, ICP did reach $25 \mathrm{mmHg}$ in the TBI group, which led to increased BBB permeability in addition to abnormalities in the cerebral hemodynamic parameters, indicating successful TBI establishment in the rabbits.

Compared with conventional non-contrast CT, CT perfusion directly reflects the dynamic changes in circulation, providing values for important hemodynamic parameters including rCBF, rCBV and MTT in the process (23-25). Following TBI induction, the decline in $\mathrm{rCBF}$ may trigger cerebrovascular autoregulation, which increases rCBV and maintains blood flow by cerebral vasodilation (26). However, in the present study, a significant decline in CBV was detected in early TBI, implying dysfunction of cerebrovascular autoregulation in rabbits following TBI.

Decompressive craniectomy is commonly applied for the management of acute TBI in the clinic $(27,28)$. However, the efficacy of cranial decompression in improving outcome and reducing disability after TBI therapy is controversial. Although most of the existing evidence indicates that cranial decompression is generally favorable in terms of TBI patient survival (13-16). one previous study demonstrated that such treatment may lead to an unfavorable outcome due to complications caused by intraoperative brain extrusion (17). Controlled decompression differs from common decompression in terms of the procedure and strategy applied for the intraoperative release of ICP. It has been reported that controlled decompression carries advantages in reducing the incidence of ischemic reperfusion injuries and acute postoperative cerebral infarction (29). In the present study, the changes in cerebral hemodynamics between common decompression (rapid release of ICP) and controlled decompression (step-by-step gradual release of ICP) in TBI animals were compared. It was found that neither common nor controlled decompression could reverse the reduced blood flow in cerebral tissues or the increase in BBB permeability following TBI induction. This phenomenon might be attributed to dysfunction in the automatic cerebrovascular adjustment system. Under normal conditions, decreases in $\mathrm{CBF}$ triggers the vasodilatation of cerebral vessels to increase $\mathrm{CBV}$ to maintain adequate blood flow in brain tissues (30). In the present study, a decline in CBV after TBI was observed, which suggested a dysfunctional cerebrovascular adjustment system in early TBI. By contrast, damages to the BBB may also result in defective cerebrovascular adjusting and neurovascular coupling (31). Compared with common decompression, the use of controlled decompression was more effective for reversing low perfusion and extending MTT, but not to baseline levels. As the MTT value is affected by CBF, both common and controlled decompression induced reductions in $\mathrm{CBF}$, which may lead to the extension of MTT. It is possible that controlled decompression may prevent circulatory abnormalities by improving cerebrovascular autoregulation in rabbits following TBI injury. In addition, data from the present study revealed that the $\mathrm{rCBF}$ value was further reduced in the bilateral temporal lobes after common decompression in the TBI-model animals. However, the change in $\mathrm{rCBV}$ in the bilateral temporal lobe and the basal ganglion varied between the TBI and TBI + common decompression groups. The basal ganglion is localized deep in the brain and has an abundant blood supply. In this study, intracranial hypertension was induced in rabbits 
by epidural compression, which exhibited more notable effects on the hemodynamic changes in the frontal and temporal lobes, while the influence on basal ganglion hemodynamics might lag behind or be relatively small. Although the underlying mechanisms remain unclear, this phenomenon may explain why decompressive craniectomy reduced ICP but did not improve the poor overall prognosis in patients with severe TBI $(27,28)$. A previous preliminary study compared the therapeutic outcomes of common decompression and controlled decompression in 128 patients afflicted with severe head injury (29). According to those findings, controlled decompression is more potent than common decompression for preventing the incidence of intraoperative acute brain swelling. Nevertheless, the application of controlled decompression in animal studies and patients is a relatively novel concept, and the efficacy of this therapeutic strategy requires further exploration

This study does carry some limitations. Firstly, a local TBI injury model was used here, and therefore the possibility that the cerebral hemodynamics may vary in other TBI models could not be ruled out. Secondly, the indices of cerebral hemodynamics were measured $30 \mathrm{~min}$ after injury, and the changes in these indices had not yet been evaluated for a more prolonged time period. Thirdly, in this study, TBI was induced by increasing the ICP to over $25 \mathrm{mmHg}$, and the changes in cerebral hemodynamics under different ICP values have not been determined. Lastly, future studies are still required for exploring the mechanism by which controlled decompression alleviates the circulatory abnormalities induced by TBI injury.

In conclusion, the present study demonstrated that CT perfusion could be used to analyze local changes in cerebral hemodynamics after TBI induction in rabbits. TBI induced significant reductions in $\mathrm{rCBF}$ and $\mathrm{rCBV}$, prolonged MTT and greatly increased BBB permeability in rabbits. Controlled decompression was more effective compared with common decompression at preventing these abnormalities in cerebral hemodynamics following TBI injury induction. These findings provide valuable insights for understanding the use of CT perfusion to evaluate therapeutic outcomes and cerebral hemodynamics in patients with TBI.

\section{Acknowledgements}

Not applicable.

\section{Funding}

The present study was supported by The Science Foundation of Wuxi Municipal Commission of Health and Family Planning in China (grant no. MS201527 to KFC).

\section{Availability of data and materials}

The datasets used and analyzed during the present study are available from the corresponding author upon reasonable request.

\section{Authors' contributions}

KFC and YHW designed the experiments; KFC and FHD performed the experiments; GXL and JRD analyzed the data; KFC wrote the paper.

\section{Ethics approval and consent to participate}

The animal study was approved by the Animal Ethics Committee of Wuxi Clinical College, Anhui Medical University (Wuxi, China).

\section{Patient consent for publication}

Not applicable.

\section{Competing interests}

The authors declare that they have no competing interests.

\section{References}

1. Iaccarino C, Carretta A, Nicolosi F and Morselli C: Epidemiology of severe traumatic brain injury. J Neurosurg Sci 62: 535-541, 2018.

2. Blennow K, Brody DL, Kochanek PM, Levin H, McKee A, Ribbers GM, Yaffe K and Zetterberg H: Traumatic brain injuries. Nat Rev Dis Primers 2: 16084, 2016.

3. Teasdale G, Murray G, Parker L and Jennett B: Adding up the glasgow coma score. Acta Neurochir Suppl (Wien) 28: 13-16, 1979.

4. Jiang JY and Chinese Head Trauma Study Collaborators: Head trauma in China. Injury 44: 1453-1457, 2013.

5. Oddo M, Levine JM, Mackenzie L, Frangos S, Feihl F, Kasner SE, Katsnelson M, Pukenas B, Macmurtrie E, Maloney-Wilensky E, et al: Brain hypoxia is associated with short-term outcome after severe traumatic brain injury independently of intracranial hypertension and low cerebral perfusion pressure. Neurosurgery 69: 1037-1045, 2011.

6. Farahvar A, Gerber LM, Chiu YL, Härtl R, Froelich M, Carney N and Ghajar J: Response to intracranial hypertension treatment as a predictor of death in patients with severe traumatic brain injury. J Neurosurg 114: 1471-1478, 2011.

7. Kaloostian P, Robertson C, Gopinath SP, Stippler M, King CC, Qualls C, Yonas H and Nemoto EM: Outcome prediction within twelve hours after severe traumatic brain injury by quantitative cerebral blood flow. J Neurotrauma 29: 727-734, 2012.

8. Rostami E, Engquist H and Enblad P: Imaging of cerebral blood flow in patients with severe traumatic brain injury in the neurointensive care. Front Neurol 5: 114, 2014.

9. Lim D, Lee SH, Kim DH, Choi DS, Hong HP, Kang C, Jeong JH, Kim SC and Kang TS: The possibility of application of spiral brain computed tomography to traumatic brain injury. Am J Emerg Med 32: 1051-1054, 2014.

10. Servadei F, Nasi MT, Giuliani G, Cremonini AM, Cenni P, Zappi D and Taylor GS: CT prognostic factors in acute subdural haematomas: The value of the 'worst' CT scan. Br J Neurosurg 14: $110-116,2000$

11. Douglas DB, Chaudhari R, Zhao JM, Gullo J, Kirkland J, Douglas PK, Wolin E, Walroth J and Wintermark M: Perfusion imaging in acute traumatic brain injury. Neuroimaging Clin $\mathrm{N}$ Am 28: 55-65, 2018

12. Bendinelli C, Bivard A, Nebauer S, Parsons MW and Balogh ZJ: Brain CT perfusion provides additional useful information in severe traumatic brain injury. Injury 44: 1208-1212, 2013.

13. Brazinova A, Mauritz W, Leitgeb J, Wilbacher I, Majdan M, Janciak I and Rusnak M: Outcomes of patients with severe traumatic brain injury who have Glasgow Coma Scale scores of 3 or 4 and are over 65 years old. J Neurotrauma 27: 1549-1555, 2010.

14. Bell RS, Mossop CM, Dirks MS, Stephens FL, Mulligan L, Ecker R, Neal CJ, Kumar A, Tigno T and Armonda RA: Early decompressive craniectomy for severe penetrating and closed head injury during wartime. Neurosurg Focus 28: E1, 2010.

15. Gouello G, Hamel O, Asehnoune K, Bord E, Robert R and Buffenoir K: Study of the long-term results of decompressive craniectomy after severe traumatic brain injury based on a series of 60 consecutive cases. ScientificWorldJournal 2014: 207585, 2014. 
16. Charry JD, Rubiano AM, Nikas CV, Ortíz JC, Puyana JC, Carney N and Adelson PD: Results of early cranial decompression as an initial approach for damage control therapy in severe traumatic brain injury in a hospital with limited resources. J Neurosci Rural Pract 7: 7-12, 2016.

17. Quintard H,Lebourdon X, Staccini P and Ichai C: Decompression surgery for severe traumatic brain injury (TBI): A long-term, single-centre experience. Anaesth Crit Care Pain Med 34: 79-82, 2015.

18. Chen WL, Yang LK and Kuang H: Establishment of posttraumatic acute diffuse brain swelling with sinus balloon compression method in rabbits. Chin J Trauma 8: 753-757, 2015.

19. Gungormus M and Kaya O: Evaluation of the effect of heterologous type I collagen on healing of bone defects. J Oral Maxillofac Surg 60: 541-545, 2002.

20. Bragin DE, Statom GL, Yonas H, Dai X and Nemoto EM: Critical cerebral perfusion pressure at high intracranial pressure measured by induced cerebrovascular and intracranial pressure reactivity. Crit Care Med 42: 2582-2590, 2014.

21. Yan LZ, Sheng LY and Bing C: Changes of the transcranial Doppler spectrum wave form in the model of acute intracranial hypertension in rabbits. Bulletin Hunan Medical Univ 27: 441-444, 2002.

22. Donnelly J, Czosnyka M, Harland S, Varsos GV, Cardim D, Robba C, Liu X, Ainslie PN and Smielewski P: Cerebral haemodynamics during experimental intracranial hypertension. J Cereb Blood Flow Metab 37: 694-705, 2017.

23. Honda M, Ichibayashi R, Yokomuro H, Yoshihara K, Masuda H, Haga D, Seiki Y, Kudoh C and Kishi T: Early cerebral circulation disturbance in patients suffering from severe traumatic brain injury (TBI): A Xenon CT and perfusion CT study. Neurol Med Chir (Tokyo) 56: 501-509, 2016.

24. Pan J, Zhang J, Huang W, Cheng X, Ling Y, Dong Q and Geng D: Value of perfusion computed tomography in acute ischemic stroke: Diagnosis of infarct core and penumbra. J Comput Assist Tomogr 37: 645-649, 2013.
25. Thierfelder KM, Sommer WH, Baumann AB, Klotz E, Meinel FG, Strobl FF, Nikolaou K, Reiser MF and von Baumgarten L: Whole-brain CT perfusion: Reliability and reproducibility of volumetric perfusion deficit assessment in patients with acute ischemic stroke. Neuroradiology 55: 827-835, 2013.

26. Powers WJ, Grubb RL Jr and Raichle ME: Physiological responses to focal cerebral ischemia in humans. Ann Neurol 16: 546-552, 1984

27. Barthelemy EJ, Melis M, Gordon E, Ullman JS and Germano IM: Decompressive craniectomy for severe traumatic brain injury: A systematic review. World Neurosurg 88: 411-420, 2016.

28. Cooper DJ, Rosenfeld JV, Murray L, Arabi YM, Davies AR, D'Urso P, Kossmann T, Ponsford J, Seppelt I, Reilly P, et al: Decompressive craniectomy in diffuse traumatic brain injury. $\mathrm{N}$ Engl J Med 364: 1493-1502, 2011.

29. Wang Y, Wang C, Yang L, Cai S, Cai X, Dong J, Zhang J and Zhu J: Controlled decompression for the treatment of severe head injury: A preliminary study. Turk Neurosurg 24: 214-220, 2014.

30. Toth P, Szarka N, Farkas E, Ezer E, Czeiter E, Amrein K, Ungvari Z, Hartings JA, Buki A and Koller A: Traumatic brain injury-induced autoregulatory dysfunction and spreading depression-related neurovascular uncoupling: Pathomechanisms, perspectives, and therapeutic implications. Am J Physiol Heart Circ Physiol 311: H1118-H1131, 2016.

31. Moretti R, Pansiot J, Bettati D, Strazielle N, Ghersi-Egea JF, Damante G, Fleiss B, Titomanlio L and Gressens P: Blood-brain barrier dysfunction in disorders of the developing brain. Frontiers Neuroscience 9: 40, 2015.

This work is licensed under a Creative Commons Attribution-NonCommercial-NoDerivatives 4.0 International (CC BY-NC-ND 4.0) License. 